\title{
Perceptual Speech Processing and Phonetic Feature Mapping for Robust Vowel Recognition
}

\author{
Linkai Bu, Member, IEEE, and Tzi-Dar Chiueh, Member, IEEE
}

\begin{abstract}
In this paper, we propose the perceptual speech processing and the phonetic feature mapping, which are inspired by human auditory perceptual characteristics. The proposed perceptual speech processing is based on three perceptual characteristics and consists of three independent processing steps: masking effect, minimum audible field renormalization, and mel-scale resampling. They remove unperceptible spectral components, and adjust magnitude and frequency scales of speech spectra, respectively. We apply these three processing steps to speech spectrum sequentially to generate a new speech signal representation called perceptual spectrum. For Mandarin vowel recognition, nine representative vowels are selected as references and similarity measures to these reference spectra, called phonetic features, are then generated from perceptual spectrum. These phonetic features then serve as speech parameters in a continuous HMM-based recognition stage. With these two techniques, high recognition accuracy on Mandarin vowel phonemes has been achieved. Further experiments confirm that significant improvement on recognition robustness with respect to speaker variation and noise contamination can be also obtained.
\end{abstract}

Index Terms-Phonetic feature, robust speech recognition, speech perception.

\section{INTRODUCTION}

$\mathbf{N}$ OISES, distortions, and unforeseen speakers seldom cause difficulty for human to understand speech signals whereas they seriously degrade performances of automatic speech recognition (ASR) systems. Many researchers have thus made various efforts on emulating human speech perception in order to achieve human-like performance. These efforts can be divided into two categories. The first type of approaches model and rebuild the functionalities of the auditory nuclei [1]. These are exemplified by research on basilar membrane and electronic cochlea [2]-[4]. Most of this type of systems have similar structures as human auditory pathway. They implement, however, only a small fraction of perceptual functionality in the human auditory system. This is because the human auditory system is structurally very complex, consisting of many feedback paths from higher levels in the neural system. Presently, much of the structure and mechanism, especially interactions among auditory nuclei, remains unknown.

The other type of approaches resort to neural networks for circumventing difficulties encountered in speech recognition

Manuscript received November 14, 1996; revised March 12, 1999. This work was supported in part by the National Science Council, Taiwan, R.O.C., under Grant NSC85-2215-E-002-016. The associate editor coordinating the review of this manuscript and approving it for publication was Dr. Mazin Rahim.

The authors are with the Department of Electrical Engineering, National Taiwan University, Taipei, Taiwan 10617, R.O.C. (e-mail: chiueh@cc.ee.ntu.edu.tw).

Publisher Item Identifier S 1063-6676(00)01707-7. under real-life environments [5]-[7]. Some systems use neural networks to extract speech features [8], [9], while others adopt neural networks to handle the dynamic and nonlinear behaviors of speech signals [10], and still others integrate neural networks with statistical recognizers in order to combine advantages from both paradigms [11]-[14]. The most beneficial characteristics of neural networks for ASR are fault tolerance and nonlinear property [15], [16]. However, current neural network based ASR systems fail to exploit perceptual characteristics in human auditory system, which are conjectured to be key ingredients in robust speech recognition [17], [18].

Psychologists have tried to unlock the mystery of human speech perception by measuring aggregate responses to various stimuli. Human response to specific sounds contains clues about mechanisms of the underlying processing in human speech recognition. Many such measurements and models explaining these measurements have been reported [19], [20]. In this paper, we apply three major perceptual processing to speech Fourier spectrum and obtain a new speech representation, called perceptual spectrum. Furthermore, to reduce feature dimensionality and at the same time retain relevant information, we propose a phonetic feature mapping. In this mapping, a small set of features, called phonetic features, are generated. These new features are basically similarity measures between the input perceptual spectrum and a set of nine reference perceptual spectra.

Fig. 1 illustrates the block diagram of the proposed robust vowel recognition system. Two processing stages, the perceptual speech processing stage and the phonetic feature mapping stage, are inserted between the FFT stage and the continuous hidden Markov model (CHMM) recognizer. The perceptual processing stage contains three processing steps: masking effect, minimum audible field (MAF) renormalization, and mel-scale resampling. Based on the perceptual spectrum, phonetic features are generated in the following phonetic feature mapping stage. The mapping is inherently analogous to the partial recognition of human speech perception. It "classifies" the speech spectrum in a soft-decision manner. Unlike the conventional classification that categorizes input speech spectrum into one and only one class (unary coding), it evaluates similarities of the input speech spectrum to several reference spectra (soft coding). These phonetic features then serve as speech parameters in the final CHMM-based recognition stage.

The rest of this paper is organized as follows. Section II describes three perceptual characteristics adopted in the perceptual spectrum and illustrates their effects by an example. The principle of phonetic feature mapping is next introduced in Section III. This section also demonstrates, through an example, how the 


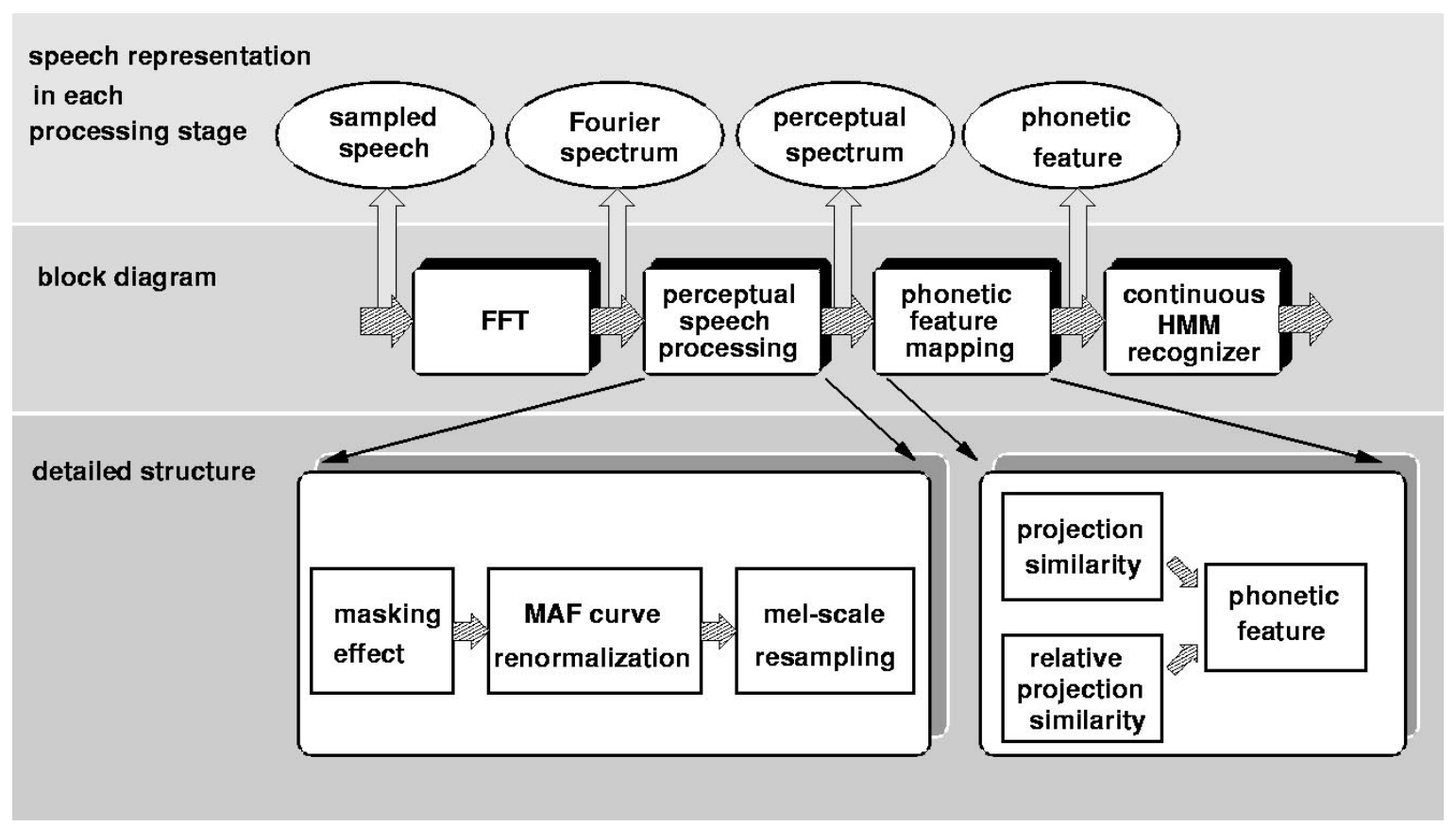

Fig. 1. Block diagram of the proposed speech recognition system.

phonetic features better discriminate two similar vowels. Section IV evaluates the two proposed techniques through a series of recognition experiments. According to the experiment results, the relationship among discernibility of speech parameters, recognition accuracy, and robustness in recognition are discussed in Section V. Section VI summarizes and concludes this paper.

\section{Perceptual Spectrum}

Perceptual spectrum representation of speech signals is based on three perceptual characteristics: masking effect, minimum audible field (MAF), and mel-scale. Masking effect refers to the phenomenon that certain sound becomes inaudible when there are other louder sounds that are both temporally and spectrally adjacent. This effect is further divided into simultaneous, forward, and backward masking [19], [20]. Several speech compression techniques have also taken advantage of the masking effect to reduce distortion in the coding process [21]-[24]. Fig. 2 plots a masker (the solid curve) generated by an $1 \mathrm{kHz}, 80 \mathrm{~dB}$ pure tone (the small circle), and all tones below the masker can not be perceived by human. For a speech spectrum, each frequency component is deemed as a pure tone and thus has an individual masker, which has identical shape as the one in Fig. 2 except for some vertical translation according to the intensity of that frequency component. The final masker $m(f)$ of the whole spectrum is then given by

$$
m(f)=\max _{i} \psi^{(i)}(f)
$$

where $\psi^{(i)}(f)$ is the masker of the $i$ th frequency component. Finally, all frequency components below the masker $m(f)$ are removed from the spectrum.

Fig. 3 plots the equal loudness curves (the solid curves) and the MAF curve (the dashed curve) [19]. All points in an equal

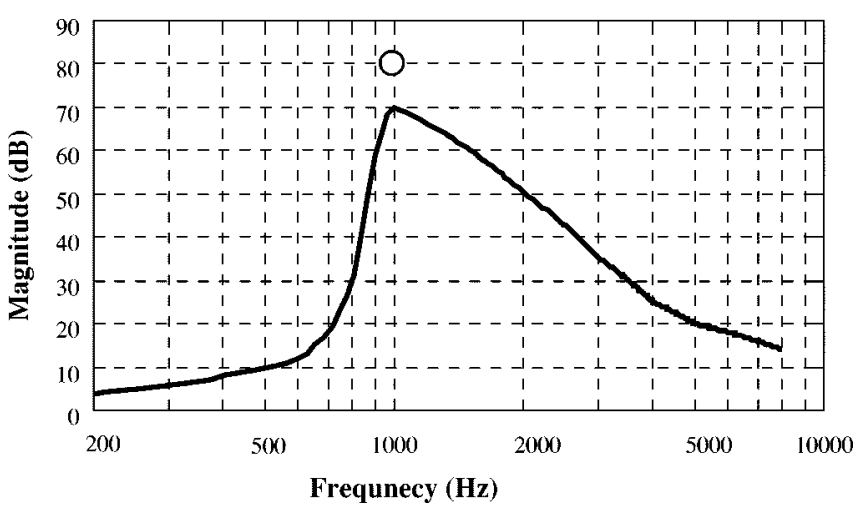

Fig. 2. The masker (the solid curve) generated by a $1 \mathrm{kHz}, 80 \mathrm{~dB}$ pure tone (the small circle).

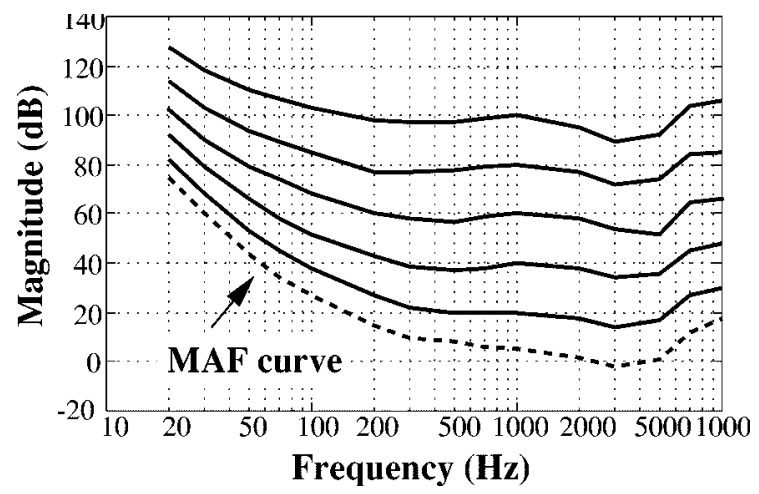

Fig. 3. Equal loudness curves and the MAF curve. Tones lying in the same curve are subjectively of equal loudness.

loudness curve are perceptually of the same loudness to human beings [25]. The sound magnitude threshold versus frequency is called the minimum audible field (MAF) curve, below which the sound signals are too weak for human to perceive. Note that 
sensation of loudness depends nonlinearly on signal power intensity [26], so exact subjective loudness computation involves time-consuming table-look-up operations of all the equal loudness curves. For convenience of implementation, we chose to renormalize magnitudes of all frequency components according to only the MAF curve

$$
l(f)=s(f)-\xi(f)
$$

where

$l(f)$ loudness at frequency $f$;

$s(f)$ intensity at frequency $f$;

$\xi(f)$ MAF value at frequency $f$.

Note the MAF curve renormalization is different from pre-emphasis of speech signals [27] since the MAF curve deemphasizes both the low- and high-frequency components while the pre-emphasis technique deemphasizes only the low-frequency component.

Finally, frequency scale also needs adjustment according to subjective pitch sensation. The mel-scale, as described in [28], is obtained by

$$
\mu=2595 \cdot \log \left(1+\frac{f}{700}\right)
$$

where $f$ is the signal frequency and $\mu$ denotes the mel-scale. Physiology studies also found similar properties in the cochlea basilar membrane [29], called the Greenwood's function.

To sum up, perceptual spectrum is obtained by applying the following three procedures sequentially to the Fourier spectrum (see Fig. 1):

1) Remove frequency components shadowed by louder neighboring components according to the final maskers in the previous and current frames.

2) Renormalize magnitude of each frequency component according to the MAF curve.

3) Translate components to mel-scale by resampling.

The three processing steps are arranged in such order for numerical convenience and their order may be different from that in the auditory pathway [26].

Fig. 4 demonstrates the effects of these three perceptual processing procedures. Fig. 4(a) shows the Fourier spectrum of the Mandarin vowel " $i$," and Fig. 4(b) displays the result after applying the masking effect. Note that the masking effect eliminates most frequency components between $400 \mathrm{~Hz}$ and 2 $\mathrm{kHz}$, which contain no formats and are irrelevant to later vowel recognition. Fig. 4(c) is the result of the MAF processing. One can see that with magnitude renormalization according to the MAF curve, low- and high-frequency components are attenuated. After the mel-scale resampling, the perceptual spectrum of the vowel " $i$ " is shown Fig. 4(d). Note that low-frequency components, where most vowel information is carried, are resampled more finely and thus are spread to a larger extent. The final perceptual spectrum preserves only spectral envelope since that alone conveys information about the shape of the vocal tract. This also removes pitch information, which is not essential to vowel recognition. Robustness of the perceptual spectrum to noises and speaker variation will be confirmed by several recognition experiments in Section IV.
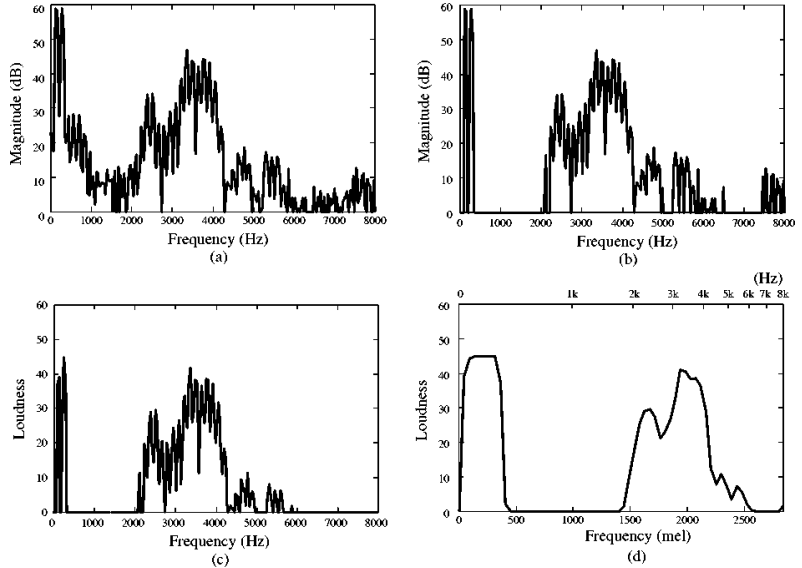

Fig. 4. Example of perceptual speech processing. (a) Fourier spectrum of the Mandarin vowel "i," (b) after masking effect process, (c) after MAF curve renormalization, and (d) after mel-scale resampling (perceptual spectrum).
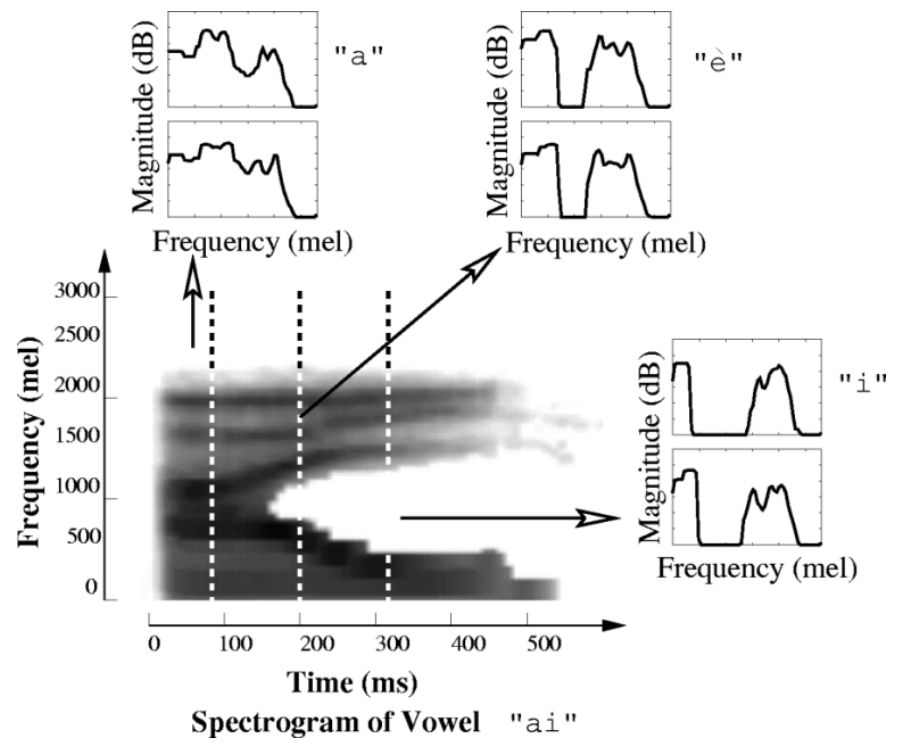

Fig. 5. Spectrogram of Mandarin vowel "ai."

\section{Phonetic Feature MAPPING}

This section presents the phonetic feature mapping which generates new phonetic features based on similarity measures to a set of reference spectrum vectors. When humans articulate, air is pushed out from the lungs and excites the vocal cord. The vocal tract then filters the pressure wave and effectively shapes its spectrum. For some vowels, the vocal tract shape remains unchanged throughout the articulation, so the corresponding spectral shapes are also stationary in time. For other vowels, the articulation begins with a vocal tract shape, gradually changes, and then settles down to another shape. Similar transitions in the corresponding spectrogram can also be observed.

For stationary vowels, spectral shape is the only cue for phoneme discrimination and their spectra are adopted as reference spectra in the phonetic feature mapping. On the contrary, nonstationary vowels contain two or three segments of reference vowels and transitions between these vowels. An example spectrogram of the nonstationary Mandarin vowel "ai" is shown in Fig. 5. It starts with a spectrum similar to 
TABLE I

All 37 MANDARIN Vowel PHONEMES AND THE NINE REFERENCE PHONEMES

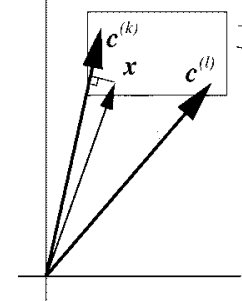

(a)

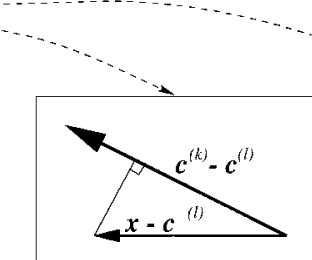

(b)

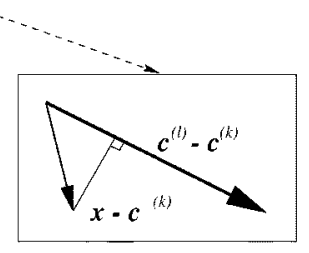

(c)

Fig. 6. (a) Reference vectors $\boldsymbol{c}^{(k)}$ and $\boldsymbol{c}^{(l)}$ and input $\boldsymbol{x}$, (b) projection of $\boldsymbol{x}-$ $c^{(l)}$ on $\boldsymbol{c}^{(k)}-c^{(l)}$, and (c) projection of $\boldsymbol{x}-\boldsymbol{c}^{(k)}$ on $\boldsymbol{c}^{(l)}-\boldsymbol{c}^{(k)}$.

vowel "a," then shifts to one similar to vowel "è," and finally settles down to that of vowel "i."

Based on the observation that nine stationary vowels form the basis of all 37 Mandarin vowels, we chose these nine stationary vowels (see Table I) as the reference vowels. Their spectra are denoted as $\boldsymbol{c}(k), k=1,2, \ldots, 9$. Each $\boldsymbol{c}$ is a 64-dimensional spectrum vector computed by averaging all frames of 64-point spectrum of a particular reference vowel in the training set. Also denote the standard deviation of dimension $i$ in the ensemble corresponding to reference vector $k$ as $\sigma_{i}^{(k)}$.

To reduce the dimensionality of the data fed to the HMM recognition stage, we propose the phonetic feature mapping, which generates nine features from a 64-dimensional spectrum vector. This mapping first computes the similarities of an input spectrum to the nine reference spectrum vectors. It then computes another set of 72 relative similarities between the input spectrum and 72 pairs of reference spectrum vectors. The final set of nine phonetic features are obtained by combining these 81 similarities through a method to be explained later. Unlike conventional classification process that categorizes the input spectrum into one of the nine reference spectra, the phonetic features quantitatively gauge the shape of input spectrum (or the shape of the vocal tract) against the nine reference spectra.

The phonetic feature mapping is a process of feature extraction or dimensionality reduction through similarity measures. In the literature, many similarity measures between two vectors have been reported [30], such as inner product, angular cosine, Euclidean distance, sum of absolute difference, self-organizing feature map (SOFM) [6], etc. A proper similarity measure for two speech spectrum vectors should take into account both angular proximity of the two vectors and length of the input vector. This is because speech spectra varies across a wide dynamic range and frames with relatively larger energy should have more weight on the decision. Preliminary experiments suggest that most of the well-known similarity measures are not suitable. We found that only projection-based similarity measures achieve acceptable performance. The proposed phonetic features are derived from two different projection measures: projection similarity and relative projection similarity.

\section{A. Projection Similarity}

The projection similarity is proportional to the projection of the input vector $\boldsymbol{x}$ along the direction of reference vector $\boldsymbol{c}^{(k)}$ with some weighting. The projection similarity of $\boldsymbol{x}$ on $\boldsymbol{c}^{(k)}$ is given by

$$
a^{(k)}=\sum_{i=1}^{64} w_{i}^{(k)} \cdot x_{i} \cdot \frac{c_{i}^{(k)}}{\left\|c^{(k)}\right\|}, \quad k=1, \cdots, 9,
$$

where $\left\|\boldsymbol{c}^{(k)}\right\|$ is $\sqrt{\sum_{i=1}^{64}\left(c_{i}^{(k)}\right)^{2}}$ and $w_{i}^{(k)}$ is the statistical weighting factor given by

$$
w_{i}^{(k)}=\frac{c_{i}^{(k)} / \sigma_{i}^{(k)}}{\sum_{i=1}^{64} c_{i}^{(k)} / \sigma_{i}^{(k)}}, \quad i=1, \cdots, 64, k=1, \cdots, 9,
$$

where $\sigma_{i}^{(k)}$ is the standard deviation of dimension $i$ in the ensemble corresponding to the $k$ th reference vowel. Note that in the weighting factor, $\sigma_{i}^{(k)}$ serves as a constant that make all dimensions in all nine reference vectors of the same variance, which is a common technique in pattern recognition [31]. The $c_{i}^{(k)}$ term in the weighting factor acts to emphasize spectral components with larger magnitude. At last the set of weights that corresponds to each reference vector are normalized.

The projection similarities are in general good enough, however, in cases where the input is close to several similar phonemes, clear-cut decisions are difficult to reach. This is because the projection similarities of the input to those similar reference vowels are all large. For example, vowel " $i$ " and "iu" are similar in spectral shape. An input vector which has a large projection similarity to reference vowel " $i$ " generally also has a large projection similarity to "iu." It is hard to discriminate among these two vowels by projection similarity alone. In order to improve the discernibility among similar reference vowels, we introduce another set of similarities.

\section{B. Relative Projection Similarity}

Since the projections of an input vector along two similar reference vectors carry little information on categorization of the input, we propose to extract only the critical spectral components using the relative projection similarity. For clarity of explanation and without loss of generality, assume two-dimensional vectors. Consider the case shown in Fig. 6(a), where $\boldsymbol{c}^{(k)}$ and $\boldsymbol{c}^{(l)}$ are two similar reference vectors and input $\boldsymbol{x}$ is somewhat closer to $c^{(k)}$. The difference between $c^{(k)}$ and $\boldsymbol{c}^{(l)}\left(\boldsymbol{c}^{(k)}-\right.$ $\left.c^{(l)}\right)$ is deemed critical for categorization. In Fig. 6(b) and (c), one finds that the projection of $\boldsymbol{x}-\boldsymbol{c}^{(l)}$ on $\boldsymbol{c}^{(k)}-\boldsymbol{c}^{(l)}$ is larger than the projection of $x-c^{(k)}$ on $\boldsymbol{c}^{(l)}-\boldsymbol{c}^{(k)}$ and their difference more notable than the difference between the projections of $\boldsymbol{x}$ on $\boldsymbol{c}^{(k)}$ and on $\boldsymbol{c}^{(l)}$. Following the above observation, we first compute the statistically-weighted projection of the input vector $\boldsymbol{x}$ on $\boldsymbol{c}^{(k)}$ with respect to $\boldsymbol{c}^{(l)}$ as

$$
\begin{gathered}
q^{(k, l)}=\sum_{i=1}^{64} v_{i}^{(k, l)} \cdot\left(x_{i}-c_{i}^{(l)}\right) \cdot \frac{\left(c_{i}^{(k)}-c_{i}^{(l)}\right)}{\left\|\boldsymbol{c}^{(k)}-\boldsymbol{c}^{(l)}\right\|}, \\
k, l=1, \cdots, 9, l \neq k
\end{gathered}
$$


where $\left\|c^{(k)}-c^{(l)}\right\|$ is $\sqrt{\sum_{i=1}^{64}\left(c_{i}^{(k)}-c_{i}^{(l)}\right)^{2}}$ and $v_{i}^{(k, l)}$ is the normalized weighting factor given by

$$
\begin{array}{r}
v_{i}^{(k, l)}=\frac{\left|c_{i}^{(k)}-c_{i}^{(l)}\right| / \sqrt{\sigma_{i}^{(k)^{2}}+\sigma_{i}^{(l)^{2}}}}{\sum_{i=1}^{64}\left|c_{i}^{(k)}-c_{i}^{(l)}\right| / \sqrt{\sigma_{i}^{(k)^{2}}+\sigma_{i}^{(l)}}}, \\
\quad i=1, \cdots, 64, k, l=1, \cdots, 9, l \neq k .
\end{array}
$$

Note that the weighting factors again serve to emphasize those components that the two reference vectors have large differences as well as make variances in all dimensions the same. In some cases, $q^{(k, l)}$ may become negative, to control the dynamic range and without losing cues for discriminating the input, negative $q^{(k, l)}$ is set to a small positive value while positive $q^{(k, l)}$ remains unchanged. This process is called the unipolar ramp function [15].

The relative projection similarity of $\boldsymbol{x}$ on $\boldsymbol{c}^{(k)}$ with respect to $c^{(l)}\left(r^{(k, l)}\right)$ is defined as:

$$
r^{(k, l)}=\frac{q^{(k, l)}}{q^{(k, l)}+q^{(l, k)}}, \quad k, l=1, \cdots, 9, l \neq k .
$$

There are on the total $8 \times 9=72$ relative projection similarities, which, together with nine projection similarities, shall define the proposed phonetic features.

\section{Phonetic Features}

Hierarchical classification is one obvious approach to integrating projection similarities and relative projection similarities. A coarse classification based on projection similarities first selects some potential candidates with large $a^{(k)}$ 's. Then the candidates are verified using pairwise relative projection similarities. However, the coarse classification needs lots of threshold tuning to locate potential candidates, which can be both unreliable and time consuming. In addition, when speech signals are contaminated, the correct reference vector may not pass the first test, though it can be identified by relative projection similarities.

The proposed phonetic feature mapping works to integrate the projection similarity and the relative projection similarity. The new features should have the following properties.

1) Given two reference vectors with large projection similarities, they should be decided solely by the relative projection similarities.

2) Otherwise, their magnitudes depend mainly on the projection similarities.

The phonetic features are defined as:

$$
\begin{gathered}
p^{(k)}=\frac{1}{\lambda} a^{(k)}+\frac{1}{\lambda} \sum_{l=1, l \neq k}^{9}\left(r^{(k, l)} p^{(l)}-r^{(l, k)} p^{(k)}\right), \\
k=1,2, \cdots, 9
\end{gathered}
$$

where $\lambda$ is a scaling factor. Without loss of generality, consider only two reference vectors $\boldsymbol{c}^{(k)}$ and $\boldsymbol{c}^{(l)}$. Solving (2) yields the ratio of $p^{(k)}$ to $p^{(l)}$ as

$$
\frac{p^{(k)}}{p^{(l)}}=\frac{\lambda a^{(k)}+\left(a^{(k)}+a^{(l)}\right) r^{(k, l)}}{\lambda a^{(l)}+\left(a^{(k)}+a^{(l)}\right) r^{(l, k)}} .
$$

There are three possible cases: i) both $a^{(k)}$ and $a^{(l)}$ are large and have comparable magnitudes, ii) only one of $a^{(k)}$ and $a^{(l)}$ is large, and iii) both $a^{(k)}$ and $a^{(l)}$ are small. In the first case assume that $\boldsymbol{x}$ is closer to $c^{(k)}$ in the Euclidean norm, i.e., the Euclidean distance between $\boldsymbol{x}$ and $c^{(k)}$ is smaller, so $r^{(k, l)}$ is larger than $r^{(l, k)}$. If $\lambda$ is relatively small, then $p^{(k)} / p^{(l)}$ is approximately $r^{(k, l)} / r^{(l, k)}$, which is decided by $r^{(k, l)}$ and $r^{(l, k)}$

In the second case, assume $a^{(k)}$ is large, then $r^{(k, l)}$ and $r^{(l, k)}$ are close to unity and zero, respectively. $p^{(k)} / p^{(l)}$ is approximately $\left((\lambda+1) a^{(k)}+a^{(l)}\right) / \lambda a^{(l)}$, which is determined by $a^{(k)}$ and $a^{(l)}$. Finally, consider the third case that both $a^{(k)}$ and $a^{(l)}$ are small. In this case $p^{(k)}$ and $p^{(l)}$ are respectively proportional to $\lambda a^{(k)}+\left(a^{(k)}+a^{(l)}\right) r^{(k, l)}$ and $\lambda a^{(l)}+\left(a^{(k)}+a^{(l)}\right) r^{(l, k)}$ Since $a^{(k)}$ and $a^{(l)}$ are both small and $r^{(k, l)}$ and $r^{(l, k)}$ are less than unity, thus $p^{(k)}$ and $p^{(l)}$ are also small and negligible.

If we further define

$$
r^{(k, k)}=\lambda+\sum_{l=1, l \neq k}^{9} r^{(l, k)}, \quad k=1,2, \cdots, 9
$$

then (2) can be formulated in a matrix form as

$$
\begin{gathered}
{\left[\begin{array}{ccccc}
-r^{(1,1)} & r^{(1,2)} & r^{(1,3)} & \ldots & r^{(1,9)} \\
r^{(2,1)} & -r^{(2,2)} & r^{(2,3)} & \ldots & r^{(2,9)} \\
r^{(3,1)} & r^{(3,2)} & -r^{(3,3)} & \ldots & r^{(3,9)} \\
\vdots & \vdots & \vdots & \ddots & \vdots \\
r^{(9,1)} & r^{(9,2)} & r^{(9,3)} & \cdots & -r^{(9,9)}
\end{array}\right]\left[\begin{array}{c}
p^{(1)} \\
p^{(2)} \\
p^{(3)} \\
\vdots \\
p^{(9)}
\end{array}\right]} \\
=\left[\begin{array}{c}
-a^{(1)} \\
-a^{(2)} \\
-a^{(3)} \\
\vdots \\
-a^{(9)}
\end{array}\right] .
\end{gathered}
$$

Phonetic features $p^{(k)}, k=1,2, \cdots, 9$ can then be solved by multiplying the inverse of matrix on both side of the above equation.

Fig. 7 plots the phonetic feature profile of Mandarin vowel "ai." The phonetic feature to "a" has the largest value in the beginning; then the one to vowel "è" gradually increases and becomes dominant in the transition; finally, "i" replaces "è" and comes out as the largest phonetic feature. After $450 \mathrm{~ms}$, the phonetic feature to " $u$ " becomes visible. However, it is relatively short and not as conspicuous, so can be deemed as a transition.

The relative projection similarities are introduced to enhance discernibility among similar reference vowels. Fig. 8 compares the discernibility of projection similarity (without relative projection similarity) and phonetic feature to reference spectra of vowel " $i$ " and "iu." In Fig. 8(a), the horizontal axis is the projection similarity to reference spectrum " $i$ " $\left(a^{(6)}\right)$ and the vertical axis is the projection similarity to reference spectrum "iu" $\left(a^{(8)}\right)$. In Fig. 8(b), the horizontal axis and vertical axis are the phonetic features to reference spectra " $i$ " $\left(p^{(6)}\right)$ and "iu" $\left(p^{(8)}\right)$, respectively. Each point in both figures represents a 10-ms speech frame from eight speakers. The black points denote speech frames of Mandarin vowel " $i$ " while the gray points represent frames of Mandarin vowel "iu." Points above the diagonal are more similar to reference spectrum "iu" than reference spectrum "i." Contrarily, points lying below the diagonal are more analogous to the reference spectrum "i." In 

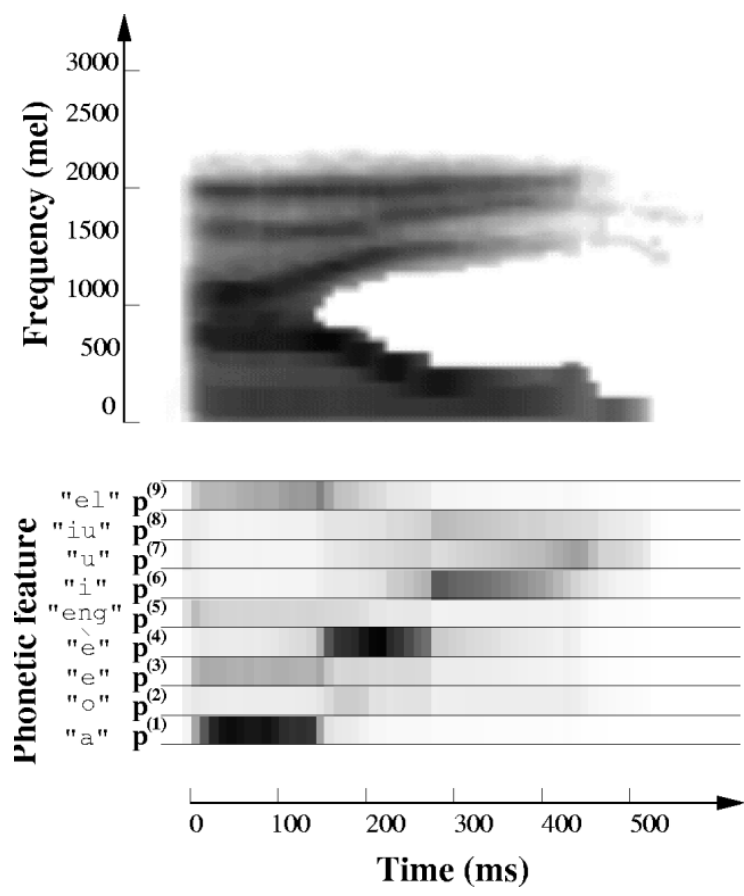

Fig. 7. Phonetic features $p^{(1)}$ to $p^{(9)}$ of Mandarin vowel "ai."

Fig. 8(a), both black points and gray points distribute around the diagonal. In Fig. 8(b), the distribution of black points and gray points shifts toward two axes, implying better discernibility for phonetic features.

The speech representation of phonetic features exhibits several perceptual properties. At first, the phonetic feature mapping is closely related to the partial recognition [1] in human speech perception. Humans perceive speech through several hierarchical partial recognition results. In the proposed ASR system, the speech spectrum is only partially recognized in the phonetic feature mapping stage since these features are intermediate results to be fed to the final HMM stage. Second, human beings ignore much irrelevant information during speech perception. In the proposed ASR system, vowels are represented by only nine phonetic features, disregarding much irrelevant information. From the experiment results in the next section, one can see that this elimination of information does not degrade the recognition accuracy, but on the contrary enhances both the recognition accuracy and robustness. Third, the number of reference vowels depends on the language. Such language dependency also occurs in human speech perception [32], [33]. Two distinct phonemes in one language may become indistinguishable for native speakers of another language, which may be caused by a lack of corresponding reference vowels in that other language.

\section{EXPERIMENTS}

This section presents a series of experiments to evaluate the proposed perceptual spectrum and the phonetic features. At first, recognition robustness subjecting to speaker variation and additive white noise is evaluated. In addition, the effectiveness of the three perceptual characteristics in perceptual speech processing is experimentally verified. Finally, properties of the two similarity measures (projection similarities and phonetic features)

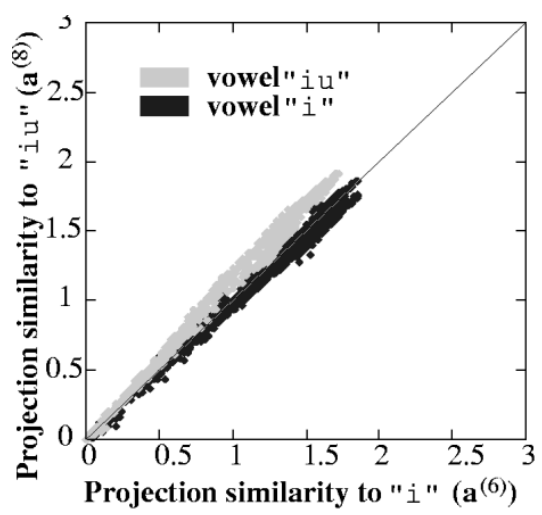

(a)

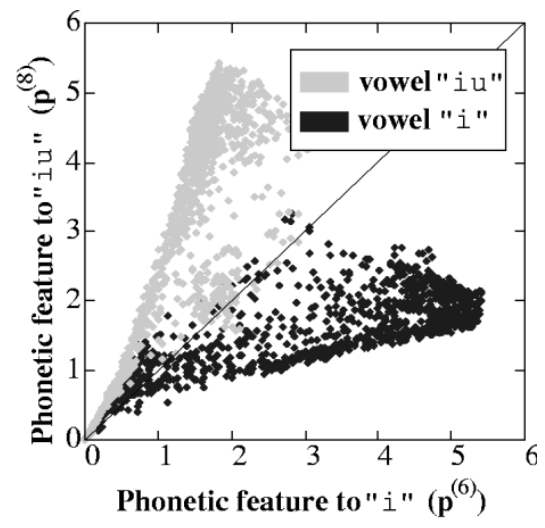

(b)

Fig. 8. Comparison of ability to discriminate two similar vowels using (a) projection similarities and (b) phonetic features.

TABLE II

GENERAL SPECIFICATIONS OF ALL EXPERIMENTS

\begin{tabular}{ll}
\hline SPEECH DATABASE \\
\hline Phonemes & all 37 Mandarin vowel phonemes \\
Speaker no. & 10 native speakers \\
Sample/speaker & 9 samples each phoneme \\
Sampling & $16 \mathrm{k} \mathrm{Hz}, 16$-bit \\
Frame Period & $10 \mathrm{~ms}$ \\
Rec. env. & general office \\
Inside test & 8 speakers \\
Outside test & 2 speakers \\
\hline RECOGNIZER & \\
\hline Type & continuous hidden Markov model \\
State no. & 9 \\
Connection & strict left-right \\
Mixture no. & 1 \\
\hline
\end{tabular}

are explored. General specifications about all experiments are listed in Table II. The corpus contains speech data from ten native speakers. Data from eight speakers are used in computing the nine reference vectors as well as in training and in the inside test. Data of the other two speakers are used in the outside test. Both unprocessed Fourier spectrum and the perceptual spectrum have been used as inputs to the phonetic feature mapping. The forgetting factor $\lambda$ is set to 0.1 in the experiments. In addition, four well known speech parameters: linear prediction coefficient (LPC), reflection coefficient (REF), cepstral coef- 
TABLE III

SPECIFICATIONS AND ABbREVIATIONS OF ALL SPEECH PARAMETERS ADOPTED IN THE EXPERIMENTS

\begin{tabular}{lll}
\hline SPEECH PARAMETER & ABBREVIATION & SIZE \\
\hline Linear prediction coefficients & LPC & order 12 \\
Reflection coefficients & REF & order 12 \\
Cepstral coefficients & CEP & order 12 \\
Mel-scale cepstral coefficients & MCEP & order 12 \\
FFT spectrum envelope & SE & 64 points \\
Perceptual spectrum & PS & 64 points \\
Phonetic feature & PF & 9 points \\
Projection similarity & PRJS & 9 points \\
\hline
\end{tabular}

TABLE IV

EXPERIMENT RESULTS OF RECOGNITION ROBUSTNESS TO SPEAKER VARIATION

\begin{tabular}{lcccc}
\hline \multirow{2}{*}{$\begin{array}{l}\text { SPEECH } \\
\text { PARAMETER }\end{array}$} & \multicolumn{2}{c}{ INSIDE RECOGNITION } & \multicolumn{2}{c}{ OUtSIDE RECOGNITION } \\
& TOP 1 & TOP 3 & TOP 1 & TOP 3 \\
\hline PF(PS) & 81.17 & 97.93 & 84.59 & 93.51 \\
PF(SE) & 75.53 & 94.16 & 72.70 & 92.16 \\
PRJS(PS) & 73.08 & 93.41 & 76.76 & 94.86 \\
PRJS(SE) & 61.22 & 85.47 & 50.27 & 81.35 \\
MCEP & 96.91 & 99.85 & 72.16 & 91.62 \\
CEP & 92.36 & 99.10 & 59.73 & 85.95 \\
REF & 86.52 & 98.53 & 46.22 & 76.76 \\
LPC & 71.50 & 91.86 & 37.84 & 62.70 \\
\hline
\end{tabular}

ficient (CEP), and mel-scale cepstral coefficient (MCEP) have been used in the recognition experiments for comparison. Their specifications and abbreviations are listed in Table III. Note that in the first six LPC- or spectrum-based approaches, either 12 or 64 features are required as opposed to only nine in the phonetic feature representation. Each of the 37 vowels are modeled by a nine-state continuous hidden Markov models (CHMM). ${ }^{1}$ Each CHMM has one mixture, strict left-right state connections, and diagonal covariance matrices. Since most Mandarin ASR systems use a natural language processing to make the final decision from the top- $N$ candidates [34], [35], we thus report top-3 recognition rates in the experiments, unless otherwise indicated. The rest of this section presents four recognition experiments: experiments on speaker variation, additive white noise, perceptual processing, and phonetic feature mapping.

\section{A. Experiments on Speaker Variation}

This experiment evaluates the recognition robustness against speaker variation by comparing the inside and outside recognition rates. The inside test uses identical speech data in both training and testing. Speech data adopted in the outside test are from speakers who are not in the training set. Experiment results of different speech parameters are listed in Table IV. The results are summarized as follows:

1) The projection similarities and the phonetic features are less sensitive to speaker variation.

2) The perceptual speech processing improves recognition accuracy.

${ }^{1}$ Diphones require large number of states. Nine-state CHMM is experimentally shown to be sufficient

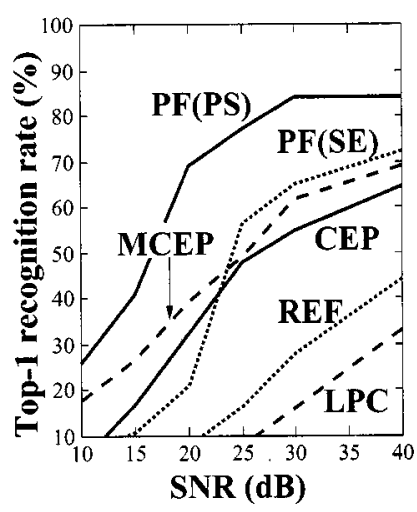

(a)

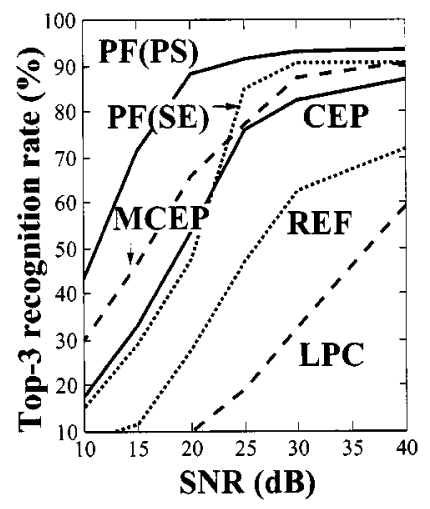

(b)
Fig. 9. Robustness against additive white noise: (a) top-1 recognition rate and (b) top-3 recognition rate.

3) The phonetic features achieve higher recognition rate than the projection similarities.

One notices that in some cases, the result of a outside test is better than that of a corresponding inside test. This is because a small corpus is used in the outside test, however, this phenomenon in no way diminishes the superiority of the perceptual spectrum and the phonetic feature mapping in speaker variation tests.

\section{B. Experiments on Additive Noise}

This experiment focuses on the robustness against additive white noise. Noisy speech data are artificially generated by adding white noise to the speech signals not in the training set. Outside recognition rates of vowel phonemes under different noise levels using various techniques are plotted in Fig. 9. The curve labeled PF(PS) extends much further to the left than all other curves. This implies that perceptual spectra based phonetic feature are more immune to additive white noise. In addition, one can find that the phonetic features of the spectrum based speech parameters (PS and SE) are more robust than the autocorrelation based parameters (LPC, REF, and CEP). Moreover, comparing curves $\mathrm{PF}(\mathrm{PS})$ and $\mathrm{PF}(\mathrm{SE})$, one notes that significant robustness improvement has been achieved by perceptual speech processing.

\section{Experiment on the Perceptual Speech Processing}

Experiment results in Fig. 9 have already shown that the perceptual speech processing can enhance the robustness. The effects of the three perceptual characteristics: masking effect, MAF curve renormalization, and mel-scale resampling are studied in this experiment. Phonetic features of the spectrum as shown in Fig. 4(a)-(d) are separately fed to a recognizer. The noisy speech recognition experiment is repeated and the results are shown in Fig. 10. Examining the figure, one finds that masking enhances both recognition accuracy and immunity against noise. The MAF renormalization improves the performance further. Finally, the mel-scale resampling slightly decreases recognition accuracy for high SNR speech but extends the recognition curve further to the left. 


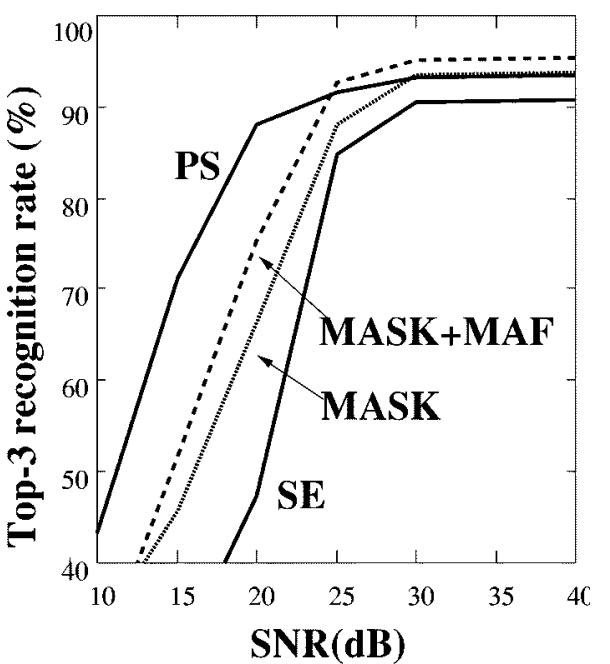

Fig. 10. Robustness improvements from the three processing steps in generating the perceptual spectrum. The curve labeled SE, MASK, MASK $+\mathrm{MAF}$, and PS are the recognition results using the phonetic features extracted from the unprocessed spectrum and spectra after successively applying the three processing steps in perceptual spectral processing, respectively.

\section{Experiment on the Phonetic Feature Mapping}

The effects of the phonetic feature mapping are shown by the experiment results plotted in Fig. 11. Perceptual spectrum, projection similarity, and phonetic feature are respectively adopted as speech parameters in three noisy speech tests. According to the curves, representing Mandarin vowels using nine similarity measures to the reference spectra (either $a^{(k)}$ or $p^{(k)}$ ) does not degrade the recognition performance but rather bring forth significant improvement. In addition, such representation reduces the size of speech parameters from 64 (spectrum) to nine. One also finds that the phonetic features are more immune to additive noise than the projection similarities. This is because the phonetic feature mapping emphasizes the critical spectral components when categorizing the input spectrum, while distortion caused by additive noise is comparatively ignored.

\section{PARAMETER DiscernibILITy, ReCOGNITION ACCURACy, AND ROBUSTNESS}

\section{A. Discernibility of the Phonetic Features}

A speech parameter is considered discernible if different phonemes can be easily discriminated in that representation. The discernibility of the phonetic features can be adjusted by $\lambda$. From (3), one sees that if $\lambda$ is large, the sum of the relative projection similarities $r^{(k, l)}$ is overwhelmed by $\lambda$. The effectiveness of the relative projection similarities becomes controllable, hence the discernibility of the phonetic features depends on the value of $\lambda$. Fig. 12 plots the distribution of two phonetic features computed from frames of the Mandarin vowel "i." Points with different gray scales represent different $\lambda$ values. Smaller $\lambda$ scatters the distribution further away from the diagonal, making these two features more discernible.

Small $\lambda$ leads to more discernible phonetic features so the recognition accuracy is improved. However, if a very small $\lambda$ is used, feature distribution of the frames in a single vowel will

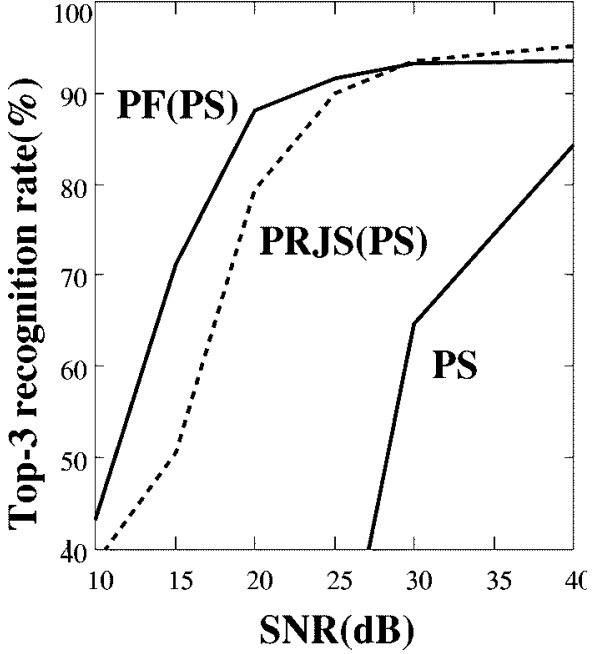

Fig. 11. Robustness comparison of three speech representations: perceptual spectrum (PS), phonetic feature of perceptual spectrum [PF(PS)], and projection similarity of perceptual spectrum [PRJS(PS)].

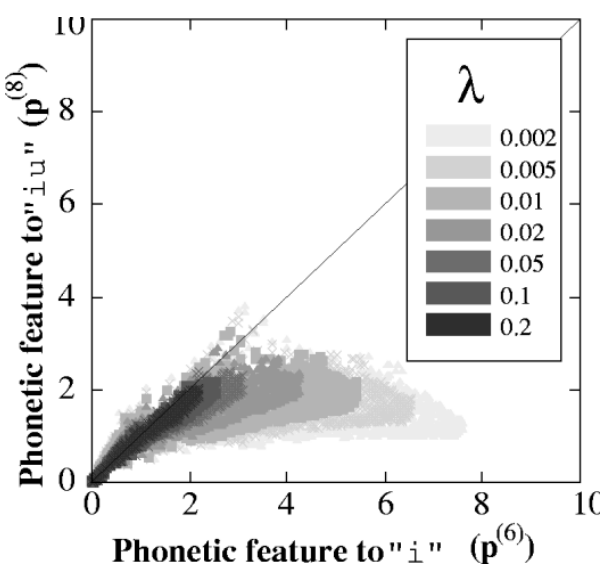

Fig. 12. Discriminating capability of the phonetic features using different values of $\lambda$. Each point represents two phonetic features $\left[p^{(6)}\right.$ and $\left.p^{(8)}\right]$ of a frame in Mandarin vowel " $i . "$

also be dispersed. Such sparse parameter distribution is hard to model by a multi-dimensional Gaussian function in the CHMM recognizer and thus results in poor recognition accuracy.

\section{B. Recognition Accuracy and Robustness}

Accuracy and robustness are two major criteria for speech parameters in ASR applications. The two attributes can be simultaneously studied using Fig. 13. The horizontal axis is the recognition rate of the inside test, and the vertical axis denotes the recognition rate under specific application requirements, such as speaker independence [Fig. 13(a)] or noisy immunity [Fig. 13(b)]. A point along the diagonal denotes speech features with robust recognition performance in the specific environment. On the other hand, points down and farther away from the diagonals denote features that are less robust. Better speech features should lie at the upper right corner indicating near perfect recognition performance. In both settings (outside speaker and noisy speech), phonetic features of the perceptual spectrum show more robustness (closer to the diagonals) than conventional parameters (LPC, REF, and CEP). 


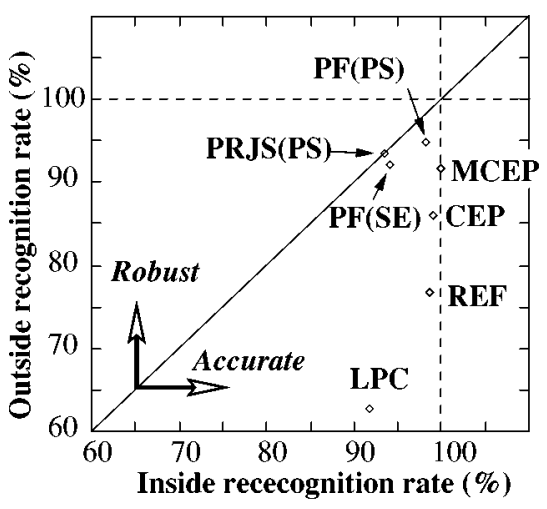

(a)

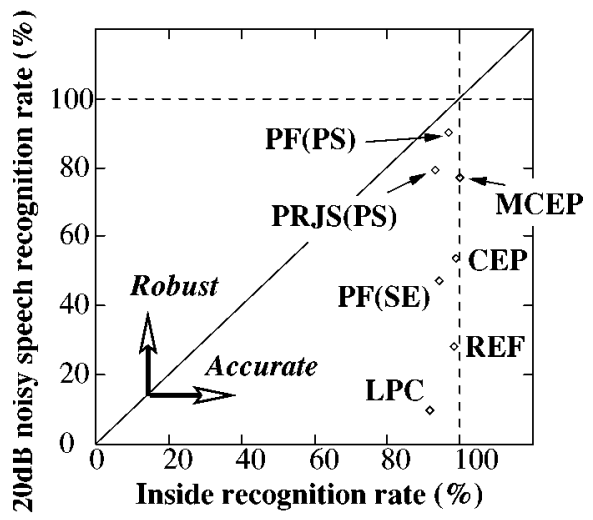

(b)

Fig. 13. Comparison of recognition accuracy and robustness using different speech parameters. Horizontal axis is the recognition rate of the inside test. Vertical axis denotes the recognition rate under nonideal environments: (a) speaker variation (outside test) and (b) noise contamination.

\section{CONCLUSIONS}

In this paper, we demonstrated the feasibility of applying human speech perceptual characteristics to enhance the recognition accuracy and robustness in Mandarin vowel recognition. In the proposed perceptual speech processing, three perceptual characteristics: masking effect, MAF-curve renormalization, and mel-scale resampling were applied to speech spectrum. The processing lays special emphasis on speech spectrum and the experiment results show that such emphasis does enhance the recognition accuracy and robustness. The phonetic feature mapping further transforms the speech representation from spectrum to phonetic features. More experiment results show that the representation of all 37 Mandarin vowel phonemes using only nine phonetic features not only increases recognition accuracy, but also enhances recognition robustness. This implies that the spectral information irrelevant to the nine reference spectra does not affect classification of Mandarin vowel phonemes. Even though the performance of the phonetic features still falls behind human auditory system, we believe this representation constitutes a solid foundation for future robust speech recognition systems.

\section{ACKNOWLEDGMENT}

The authors would like to thank the anonymous reviewers for their valuable comments and suggestions.

\section{REFERENCES}

[1] J. B. Allen, "How do humans process and recognize speech," IEEE Trans. Speech Audio Processing, vol. 2, no. 4, pp. 567-577, 1994.

[2] R. F. Lyon and C. Mead, "An analog electronic cochlea," IEEE Trans. Acoust., Speech, Signal Processing, vol. 36, pp. 1119-1134, July 1988.

[3] O. Ghitza, "Auditory models and human performance in tasks related to speech coding and speech recognition," IEEE Trans. Speech Audio Processing, vol. 2, pp. 115-132, Jan. 1994.

[4] K. Wang and S. Shamma, "Self-normalization and noise-robustness in early auditory representation," IEEE Trans. Speech Audio Processing, vol. 2, pp. 421-435, July 1994.

[5] D. P. Morgan, Neural Networks and Speech Processing. Boston, MA: Kluwer, 1991.

[6] T. Kohonen, "The self-organizing map," Proc. IEEE, vol. 78, pp. 1464-1480, Sept. 1990.

[7] R. Lippmann, "An introduction to computing with neural nets," IEEE ASSP Mag., vol. 4, pp. 4-22, Apr. 1987.

[8] E. Levin, "Hidden control neural architecture modeling of nonlinear time varying systems and its applications," IEEE Trans. Neural Networks, vol. 4, pp. 109-116, Jan. 1993.

[9] K. R. Farrell, J. Mammone, and K. T. Assaleh, "Speaker recognition using neural networks and conventional classifiers," IEEE Trans. Speech Audio Processing, vol. 2, pp. 194-205, Jan. 1994.

[10] A. J. Robinson, "An application of recurrent nets to phone probability estimation," IEEE Trans. Neural Networks, vol. 5, pp. 298-305, Mar. 1994.

[11] H. Bourlard and N. Morgan, "Continuous speech recognition by connectionist statistical methods," IEEE Trans. Neural Networks, vol. 4, pp. 893-909, Nov. 1993.

[12] C. Dugast, L. Devillers, and X. Aubert, "Combining TDNN and HMM in a hybrid system for improved continuous-speech recognition," IEEE Trans. Speech Audio Processing, vol. 2, pp. 217-223, Jan. 1994.

[13] G. Zavaliagkos, Y. Zhao, R. Schwartz, and J. Makhoul, "A hybrid segmental neural net/hidden Markov model system for continuous speech recognition," IEEE. Trans. Speech Audio Processing, vol. 2, pp. 151-160, Jan. 1994.

[14] G. Rigoll, "Maximum mutual information neural networks for hybrid connectionist-HMM speech recognition systems," IEEE Trans. Speech Audio Processing, vol. 2, pp. 175-184, Jan. 1994.

[15] J. M. Zurada, Introduction to Artificial Neural Systems. Singapore: West, 1992.

[16] L. Rabiner and B. H. Juang, Fundamentals of Speech Recognition. Englewood Cliffs, NJ: Prentice-Hall, 1993.

[17] H. Hermansky and N. Morgan, "RASTA processing of speech," IEEE Trans. Speech Audio Processing, vol. 2, pp. 578-589, Oct. 1994.

[18] J. B. Allen, "Cochlea modeling," in Proc. IEEE Int. Conf. Acoustics, Speech, Signal Processing, 1981, pp. 768-769.

[19] P. Buser and M. Imbert, Audition. Cambridge, MA: MIT Press, 1992.

[20] W. A. Yost, Fundamentals of Hearing, An Introduction. San Diego, CA: Academic, 1994

[21] N. Jayant, J. Johnston, and R. Safranek, "Signal compression based on models of human perception," Proc. IEEE, vol. 81, pp. 1385-1422, Oct. 1993.

[22] W. Chang and C. Wang, "A masking-threshold-adapted weighting filter for excitation search," IEEE Trans. Speech Audio Processing, vol. 4, pp. 124-132, Mar. 1996.

[23] N. Virag, "Speech enhancement based on masking properties of the auditory system," in Proc. IEEE Int. Conf. Acoustics, Speech, Signal Processing, 1995, pp. 796-799.

[24] A. A. Azirani, R. L. B. Jeannés, and G. Faucon, "Optimizing speech enhancement by exploiting masking properties of the human ear," in Proc. IEEE Int. Conf. Acoustics, Speech, Signal Processing, 1995, pp. 800-803.

[25] J. B. Allen, "Harvey Fletcher's role in the creation of communication acoustics," J. Acoust. Soc. Amer., vol. 99, no. 4, pp. 1825-1839, 1996.

[26] J. Allen and S. Neely, "Modeling the relation between the intensity JND and loudness for pure tones and wide-band noise," J. Acoust. Soc. Amer., vol. 102 , no. 6, pp. 3628-3646, 1997.

[27] J. D. Markel and A. H. Gray, Eds., Linear Prediction of Speech. Santa Barbara, CA: Speech Commun. Res. Lab., 1976.

[28] J. W. Picone, "Signal modeling techniques in speech recognition," Proc. IEEE, vol. 81, pp. 1215-1247, Apr. 1993.

[29] D. Greenwood, "The mel scale's disqualifying bias and a consistency of pitch-difference equisections in 1956 with equal cochlear distances and equal frequency ratios," Hearing Res., vol. 102, pp. 199-248, 1997. 
[30] T. D. Chiueh and H. K. Tsai, "Multivalued associative memories based on recurrent networks," IEEE Trans. Neural Networks, vol. 4, pp. 364-366, Mar. 1993.

[31] P. R. Krishnaiah and L. N. Kanal, Eds., Classification, Pattern Recognition, and Reduction of Dimensionality. New York: Elsevier, 1982.

[32] K. Miyawaki, W. Strange, R. Verbrugge, A. M. Liberman, J. J. Jenkins, and O. Fujimura, "An effect of linguistic experience: The discrimination of /r/ and /l/ by native speakers of Japanese and English," Percept. Psychophys., pp. 331-340, 1975.

[33] W. Strange and T. Halwes, "Confidence ratings in speech perception discrimination testing," Percept. Psychophys., pp. 182-186, 1971.

[34] J. K. Chen and F. K. Soong, "An N-best candidates-based discriminative training for speech recognition applications," IEEE Trans. Speech Audio Processing, vol. 2, pp. 206-216, Jan. 1994.

[35] L. S. Lee, C. Y. Gu, F. H. Liu, C. H. Chang, Y. H. Lin, Y. Lee, S. L. Tu, S. H. Hsieh, and C. H. Chen, "Golden Mandarin (I) - A real-time Mandarin speech dictation machine for Chinese language with very large vocabulary," IEEE Trans. Speech Audio Processing, vol. 1, pp. 158-178, Apr. 1993.

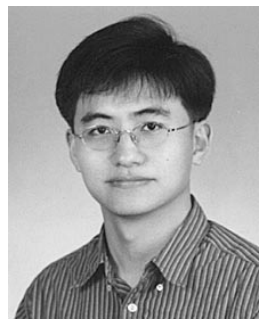

Linkai Bu (S'96-M'00) was born in 1971. He received the B.E. and Ph.D. degrees in electrical engineering from National Taiwan University, Taipei, Taiwan, R.O.C., in 1993 and 1999, respectively.

$\mathrm{He}$ is now with VIA Technologies, Inc., Taipei. His research interests include speech perception, speech recognition, signal processing, and mixed-signal VLSI design.

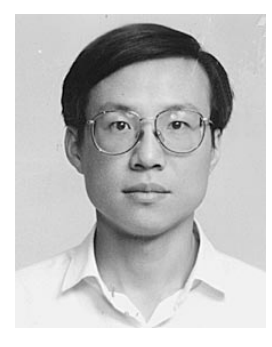

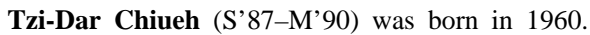
He received the B.S. degree from the Department of Electrical Engineering, National Taiwan University (NTUEE), Taipei, Taiwan, R.O.C., in 1983, and the Ph.D. degree from the California Institute of Technology, Pasadena, in 1989.

In 1989, he joined the faculty of NTUEE as an Associate Professor. He became a Full Professor in August 1994. His research interests include VLSI design of analog neuromorphic systems and digital communication systems. 